\title{
Criminologie
}

\section{Analyse spatiale des crimes commis sur la rue à l'intérieur des marchés de drogues}

\section{David Weisburd, Lorraine Green et Debra Ross}

Volume 27, numéro 1, 1994

Analyse spatiale du crime

URI : https://id.erudit.org/iderudit/017347ar

DOI : https://doi.org/10.7202/017347ar

Aller au sommaire du numéro

\section{Éditeur(s)}

Les Presses de l'Université de Montréal

ISSN

0316-0041 (imprimé)

1492-1367 (numérique)

Découvrir la revue

Citer cet article

Weisburd, D., Green, L. \& Ross, D. (1994). Analyse spatiale des crimes commis sur la rue à l'intérieur des marchés de drogues. Criminologie, 27(1), 49-67.

https://doi.org/10.7202/017347ar
Résumé de l'article

In this paper we utilize data from the Drug Market Analysis Program (DMAP) in Jersey City, New Jersey, to provide some preliminary insight into the spatial relationship between street level drug markets and crime. We begin our paper with a description of how the DMAP information system was used to define drug markets and the characteristics of the markets that were identified. We then turn to an analysis of the incidence of reported crime within drug market boundaries. We find that drug market areas include a disproportionate share of arrests and crime related emergency calls for service in Jersey City. Streets and intersections within the drug markets are also much more likely to evidence reported crime than non-drug market places. We conclude with a discussion of our findings and the implications of our research for further study of the spatial relationships between drug markets and crime.
Tous droits réservés @ Les Presses de l'Université de Montréal, 1994
Ce document est protégé par la loi sur le droit d'auteur. L'utilisation des services d’Érudit (y compris la reproduction) est assujettie à sa politique d'utilisation que vous pouvez consulter en ligne.

https://apropos.erudit.org/fr/usagers/politique-dutilisation/ 
In this paper we utilize data from the Drug Market Analysis Program (DMAP) in Jersey City, New Jersey, to provide some preliminary insight into the spatial relationship between street level drug markets and crime. We begin our paper with a description of how the DMAP information system was used to define drug markets and the characteristics of the markets that were identified. We then turn to an analysis of the incidence of reported crime within drug market boundaries. We find that drug market areas include a disproportionate share of arrests and crime related emergency calls for service in Jersey City. Streets and intersections within the drug markets are also much more likely to evidence reported crime than non-drug market places. We conclude with a discussion of our findings and the implications of our research for further study of the spatial relationships between drug markets and crime.

Au cours de la dernière décennie, le trafic de drogues illicites sur la rue et l'émergence de véritables marchés de drogues sont devenus des préoccupations majeures pour les pouvoirs publics aux États-Unis. La vente ouverte de drogues dans les rues des centres urbains a été pour la police locale et les représentants du public un rappel flagrant de l'incapacité des forces de l'ordre à prendre le contrôle des villes d'Amérique. Pour les citoyens ordinaires, la vue d'acheteurs et de vendeurs de drogues dans leur voisinage a renforcé leur sentiment de vulnérabilité face aux criminels, ainsi que leur inquiétude de voir leurs communautés envahies par le crime.

1. Cet article est basé sur une recherche financée par la subvention 90-IJ-CXK004 du National Institute of Justice. Les points de vue et opinions exprimés ici ne représentent pas nécessairement les positions ou politiques officielles du National Institute of Justice ni celles du U.S. Department of Justice. Nous aimerions remercier Al Andrews, Michael Maltz, Stephen Mastrofski, Albert J. Reiss, Jr., et Craig Uchida pour leurs conseils dans le développement du programme DMAP dans la ville de Jersey (Jersey City), et Robin Mace, Ana Lopez et Dalit Okabi pour leur collaboration au projet. Traduit de l'américain par Bernard Noël.

2. David Weisburd: The Hebrew University of Jerusalem, Institute of Criminology, Mount Scopus Jerusalem 91905, Israel; Lorraine Green : Northeastem University, College of Criminal Justice, 360 Huntington Ave, Boston, Massachusetts, Etats-Unis; Debra Ross : Rutgers University, School of Criminal Justice, 15 Washington Street, Newark, New Jersey, 07102, États-Unis. 
Le trafic de stupéfiants au niveau de la rue est donc devenu la cible $d^{*}$ un nombre croissant d'initiatives et d'innovations de la part des forces de l'ordre. Allant des traditionnelles forces d'intervention (voir Annan, 1990; Carroll, 1989, Hillsman, 1992; Kleiman et Smith, 1990) aux équipes spécialisées (voir Eck et Spelman, 1987; Kennedy, 1993) et aux programmes de réduction des nuisances dues aux stupéfiants, programmes s'appuyant sur la législation civile plutôt que sur les lois pénales sanctionnant les matières de drogues (Ferguson et Fitzsimons, 1990; Green, 1993 ; U.S. Department of Justice, 1992), les agences de police du pays entier ont concentré leurs efforts sur les endroits centraux pour la vente de stupéfiants.

Les forces de l'ordre ont développé, dans la majorité des cas, des efforts orientés contre le trafic de drogues dans la rue sans aucune référence à de l'information systématique à propos de la nature des secteurs de distribution de drogues (Hayeslip, 1989). L'abondance des recherches sur la relation liant consommation de drogues et criminalité parmi la population contrevenante réelle et potentielle (voir par exemple Altschuler et Brounstein, 1991; Chaiken et Chaiken, 1990; Goldstein, 1985 ; Innes, 1988). frappe en regard du peu d'études empiriques sur le thème de la relation spatiale entre drogues et criminalité à travers le paysage physique de la ville. En effet, selon le postulat voulant que les marchés de drogues soient au centre de l'activité criminelle, il est surprenant de constater le peu de documentation disponible sur les analyses spatiales de la relation drogue-crime. L'étude de la relation drogue-crime a presque uniquement été limitée au niveau individuel.

Dans cet article, nous utiliserons les données provenant du Drug Market Analysis Program (DMAP) de la ville de Jersey City ${ }^{3}$. Nous commencerons avec une description de la façon dont le système d'information du DMAP fut utilisé afin de définir les marchés de drogues, ainsi que les caractéristiques identifiées au sein de ceux-ci. Nous nous tournerons ensuite vers une analyse de l'incidence de la criminalité rapportée à l'intérieur des frontières des marchés de drogues identifiés. Nous constaterons que les surfaces couvrant le marché de la drogue englobent une part disproportionnée d'arrestations et de crimes, selon les appels d'urgence (911) reçus dans la ville de Jersey City. Les rues et les carrefours situés à l'intérieur des marchés de la drogue sont également beaucoup plus susceptibles de révéler des crimes rapportés que les endroits situés à l'extérieur de tels marchés. Finalement, nous discuterons des implications de notre recherche pour les études ultérieures sur la relation spatiale existante entre les marchés de drogues et la criminalité.

3. Note du traducteur : Jersey City est une ville du New Jersey située directement en face de l'île de Manhattan. Elle compte plus de 225000 habitants. 


\section{DÉFINIR LES MARCHÉS DE DROGUES : UNE APPROCHE SPATIALE}

Pour définir ce qu'est un marché de drogues, notre première tâche fut de choisir une unité d'analyse permettant d'identifier les lieux «actifs» dans le trafic des drogues. Nous avons choisi l'intersection de rue comme unité d'analyse de base. Le territoire de Jersey City comprends 1553 intersections qui définissent chacun des îlots d'habitations de la ville. Contrairement à la localisation par adresse postale souvent utilisée dans l'analyse spatiale, les intersections de la ville ne sont pas sensibles aux petites erreurs susceptibles de se produire dans la codification de la localisation des crimes. De plus, la localisation par intersection utilisée à l'intérieur du système DMAP est plus précise que la localisation par rue ou par segment de rue parce qu'elle permet de comprendre le mouvement des revendeurs de drogues d'une intersection à l'autre d'un même segment de rue.

Une fois les intersections définies sur une carte digitalisée de la ville de Jersey City, nous les avons reliées aux arrestations relatives à la vente de drogue $^{4}$, aux appels d'urgence ${ }^{5}$ et aux données provenant d'une ligne téléphonique «info-crime" spécialement établie pour le programme ${ }^{6}$. Sur un total de 1553 intersections de la ville, $41 \%$ de celles-ci étaient reliées à des activités de narcotiques par au moins une des sources de données du DMAP. Cependant, cela ne signifie pas que toutes ces intersections étaient aux prises avec un problème significatif de revente de drogues.

En effet, une arrestation isolée pour une affaire de narcotiques à une intersection n'est pas un bon indicateur de l'activité en cours. Même des arrestations multiples à un endroit spécifique durant une ou deux semaines peuvent révéler seulement un problème ponctuel. De même, une information provenant d'un citoyen ou un appel d'urgence identifiant un endroit comme un point de vente de drogues n'indique pas nécessairement qu'un tel marché prenne place. En effet, les détectives assignés aux escouades

4. Les données des ventes de narcotiques comprennent les arrestations faites entre le $1^{\text {er }}$ juin 1991 et le 30 novembre 1991. Nous avons enregistré 1844 arrestations, dont $934(51 \%)$ qui eurent lieu aux intersections et $910(49 \%)$ sur des segments de rue.

5. Les appels d'urgence dernandant de l'assistance comprennent les appels faits en rapport avec des infractions ayant trait aux stupéfiants entre le $1^{\text {er }}$ juin 1991 et le 30 novembre 1991.2196 appels furent enregistrés durant ce laps de temps. $1435(65 \%)$ d'entre eux provenaient des segments de rue et 761 (35\%) des intersections. Cette base de données d'appels ne comprend pas les appels résultant d'une arrestation ou ceux initiés par les policiers eux-mêmes.

6. Il y eu 84 informations (des "tuyaux ») concemant des affaires de narcotiques reçues par téléphone au Département de Police de la ville de Jersey du $1^{\text {er }}$ juin 1991 au 30 novembre 1991. Les téléphonistes attitrés à cette ligne info-crime reçurent de la part des citoyens des descriptions détaillées concernant des problèmes continus reliés à la drogue et entrèrent directement les détails dans un système informatisé via un *écraninformations » spécialement conçu pour le projet DMAP. 
de narcotiques savent que les citoyens prennent souvent les bandes d'adolescents pour des revendeurs de drogues. Pour qu'une intersection soit considérée comme un point de vente de drogues à l'intérieur du sys. tème DMAP, elle doit répondre aux trois critères suivants : ${ }^{7}$

1) La présence d'au moins une arrestation pour trafic de drogue durant au moins deux mois sur les six mois examinés;

2) La présence d'au moins un appel d'urgence (911) concernant une affaire de narcotiques durant deux mois séparés sur la période cible de six mois, et un minimum de sept appels au total ;

3) La présence de plusieurs appels sur la ligne info-crime de la part des citoyens.

Quelque dix pour cent des zones de carrefours de la ville de Jersey rencontrent un ou plusieurs de ces critères de sélection.

\section{L'IDENTIFICATION DES INTERSECTIONS ET SEGMENTS DE RUE ACTIFS}

L'utilisation des intersections comme unité d'analyse a comme avantage de limiter le nombre de points de vente qui auraient été éjectés de l'analyse à cause d'une mauvaise inscription de l'adresse ou à cause d'un déplacement normal du délinquant dans la rue (par exemple, il peut être arrêté à 100 mètres de l'endroit où la transaction de drogue a eu lieu). À partir de la banque de données, nous avons identifjé quelque 226 intersections et segments de rue qui peuvent être qualifiés de points de vente de drogues (sur un total de 4404 intersections et segments de rue). ${ }^{8}$

7. Le choix des critères d'identification des points de vente de drogues s'est fait après avoir testé différents modèles. Puisque nous avons choisi des critères multiples, il était nécessaire d'avoir des critères quantitatifs ( i.e. nombre d'arrestations, nombre d'appels) qui ne soient pas trop élevés.

8. Lorsque nous avons dressé la carte des intersections et des rues au sein des zones de carrefours, nous avons remarqué que seize d'cntre eux furent définis seutement par les données provenant des appels d'urgence et de la ligne info-crime. Nous pensions qu'il était improbable que ces segments de rue ou ces intersections adjacents soient de nouveaux points de distribution de drogues non répertoriés par la police : une idée qui fut largement corroborée par les officiers de police de l'escouade des narcotiques de la ville. Plutôt, nous avons pensé que des citoyens identifiaient des mouvements temporaires de contrevenants vers et hors des zones touchées par la drogue conme étant de l'activité inhérente à leur propre quartier. Dans ces cas "presque équivalents " ( « close match $»)$, nous pensions que les données d'arrestation capturaient plus fidèlement la localisation spécifique des ventes de stupéfiants. En conséquence, nous avons supprimé les carrefours et les segments de rue qui étaient identifiés seulement par les citoyens et qui étaient directement adjacents (à l'intérieur d'un quartier) aux secteurs ayant des histoires d'arrestation pour ventes de drogues. Nous avons alors coté ces sites comme des «localisations subsidiaires» aux fins d'une vérification ultérieure. 
Schéma 1

Distribution spatiale des points de vente de drogues $(n=226)$

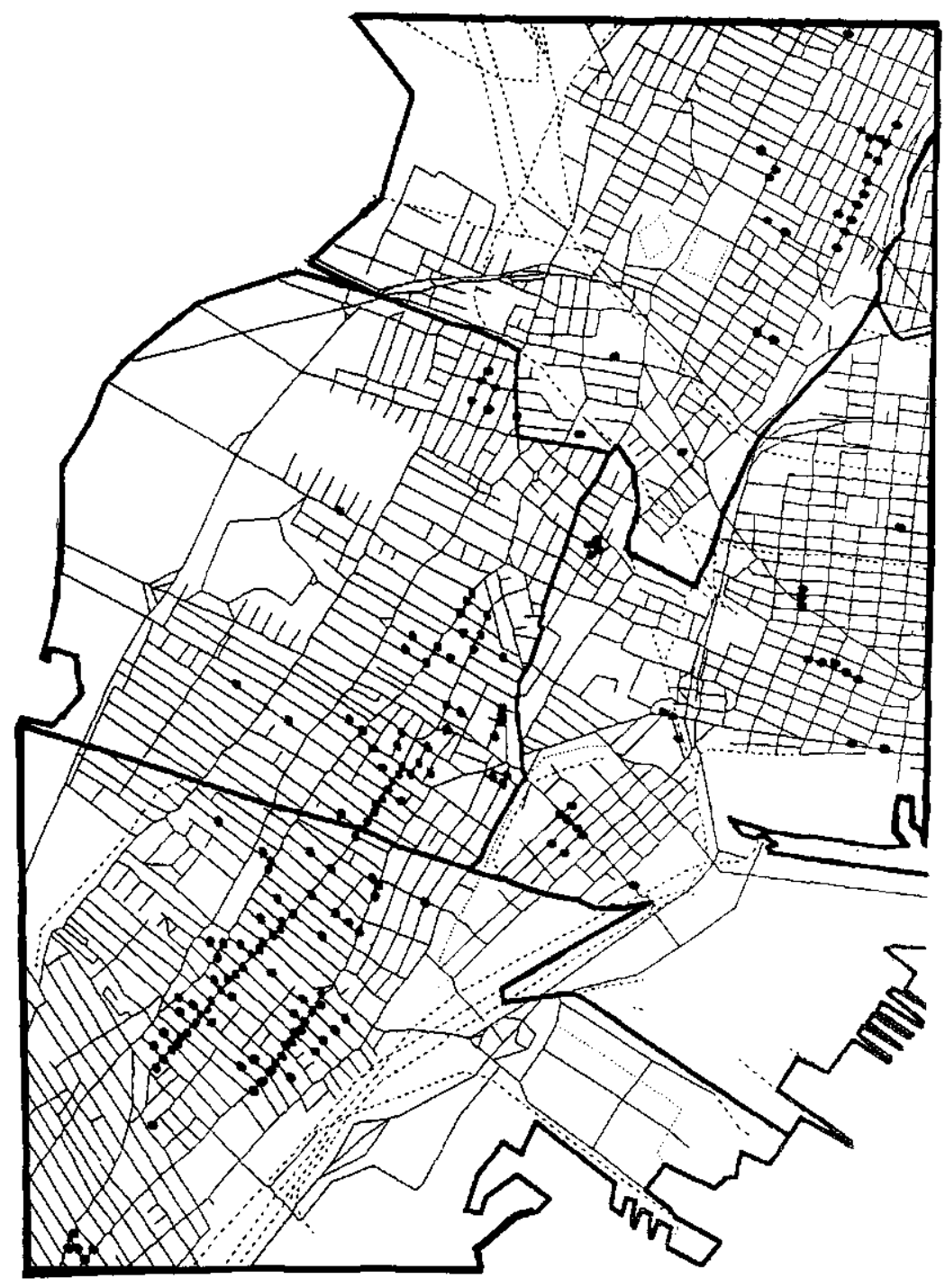


La carte finale des intersections et segments de rue actifs utilisée afin de mettre à jour les frontières des marchés de drogues est présentée au schéma 1. Visiblement, la grande majorité du territoire urbain est exempte d'activités reliées à la revente de drogues telle que nous l'avons définie. Même dans le district sud de la ville de Jersey City, lequel comprend $42 \%$ des rues et des carrefours identifiés comme actifs, la très grande majorité des segments de rue et des intersections n'apparaissent pas comme des points de vente de drogues.

Tandis que le schéma 1 fournit une bonne idée de l'endroit où les activités de revente de drogues prennent place, il illustre également une difficulté majeure à laquelle nous devons faire face lorsque nous effectuons une analyse spatiale d'acivités criminelles aussi usuelles que celle de la distribution de stupéfiants. Comment doit-on analyser les concentrations de points sur la carte d'un territoire relativement grand (voir également Maltz. Gordon et Friedman, 1991) ? Selon la concentration des intersections et des segments de rue du schéma 1, par exemple, nous pouvons conclure que la ville de Jersey City comprend trois ou quatre grands marchés de drogues, chacun couvrant 25 îlots de rues ou plus. Incidemment, nous pourrions nous demander si ces grands marchés de drogues ne pourraient être divisés en plusieurs petits marchés de drogues distincts, relativement proches les uns des autres? La section suivante de cet article traite de ces questions et présente la procédure que nous avons suivie afin de définir les frontières des marchés de drogues.

\section{IDENTIFICATION DES FRONTIËRES DES MARCHÉS DE DROGUES}

En développant un processus d'identification des marchés de drogues au niveau de la rue dans la ville de Jersey City, nous cherchions à satisfaire trois principaux critères (voir Weisburd et Green, 1993). Premièrement, le processus devait refléter autant que possible la réalité des modèles de distribution des drogues dans la ville. Deuxièmement, le processus devait pouvoir identifier les endroits spécifiques révélant un trafic de drogues important et chronique. Troisièmement, il était important que le système d'identification puisse être ultérieurement utilisé de façon routinière par les policiers.

Pour développer un portrait des marchés de drogues qui rencontrent ces critères, nous nous sommes fortement inspirés de la perception que les détectives de l'escouade des narcotiques de la ville de Jersey City avaient de la manière dont le trafic de drogues opérait au niveau de la rue dans cette ville. En effet, puisque les détectives ont une longue expérience sur cette question, il était naturel pour nous de débuter avec ce matériel. De plus, le système DMAP avait à délimiter les endroits qui pourraient devenir le point de convergence des efforts policiers. Cette dimension opérationnelle du 
DMAP a orienté le travail de recherche : les frontières des marchés de drogues devaient avoir un sens pour les policiers qui auraient la responsabilité de mener les opérations sur le terrain durant la phase expérimentale du projet.

Bien que certains quartiers de la ville aient des activités continuelles de revente de drogues sur plusieurs coins de rues, les détectives de l'escouade des narcotiques de la ville de Jersey City ne voient généralement pas ces endroits comme des zones indifférenciées. Selon eux, une série d'îlots d'habitations, ou parfois même une seule intersection, pourrait être séparée des autres selon le type de drogue qui est vendue à cet endroit. Les vendeurs tendent à négocier le même type de drogue dans le même secteur, entraînant par là une sorte de spécialisation des activités du marché. Ces vendeurs ne changent pas beaucoup de place d'un jour à l'autre, parce que s'ils le faisaient, leurs clients ne sauraient plus où les trouver, et par conséquent ne sauraient plus où trouver le type de drogue qu'ils cherchent. Tandis que nous étions initialement sceptiques face à cette hypothèse de la spécialisation des produits et vendeurs au sein d'un même marché de drogues, notre propre analyse de l'information concernant les arrestations en matière de stupéfiants la confirma généralement. Dans la ville de Jersey City, seulement $12 \%$ des segments de rue et des intersections actifs révélaient deux types de drogues différentes, ces dernières zones comptant pour plus de $30 \%$ des arrestations.

Les détectives distinguent également les secteurs comprenant plusieurs centres de marché dans un même voisinage, mais séparés les uns des autres par un petit nombre d'îlots d'habitations. Ils pensent que les vendeurs ont un fort sentiment de territorialité, lequel entraîne un degré d'isolement à l'intérieur des frontières de leurs propres marchés. Cette hypothèse a également été appuyée par nos analyses. En effet. lorsque nous avons examiné l'échantillonnage des arrestations à travers les intersections et segments de rue actifs, nous avons trouvé que seulement 15 personnes sur les 448 arrêtées plus d'une fois pour vente de stupéfiants durant l'année 1990 traversaient une intersection ou un segment de rue inactif afin de vendre dans un marché de drogues adjacent au leur. De ces 15 personnes, 6 traversaient plus de quatre segments de rues pour exercer leur métier. De plus, nos analyses ont montré que si une personne est arrêtée dans deux marchés de drogues séparés, il est plus vraisemblable qu'elle soit arrêtée dans deux districts séparés de la ville que dans un seul.

En construisant les frontières des marchés de drogues à Jersey City, nous avons suivi deux critères de base tirés de ces observations de la nature des modèles de distribution de la drogue. Premièrement, prenant en compte l'importance du type de drogue dans la détermination de l'identité d'un marché, nous avons relié les intersections et les segments de rue qui démontraient des types similaires d'activités de stupéfiants. En second, 


\section{Schéma 2}

Distribution spatiale des marchés de drogues

$(n=56)$

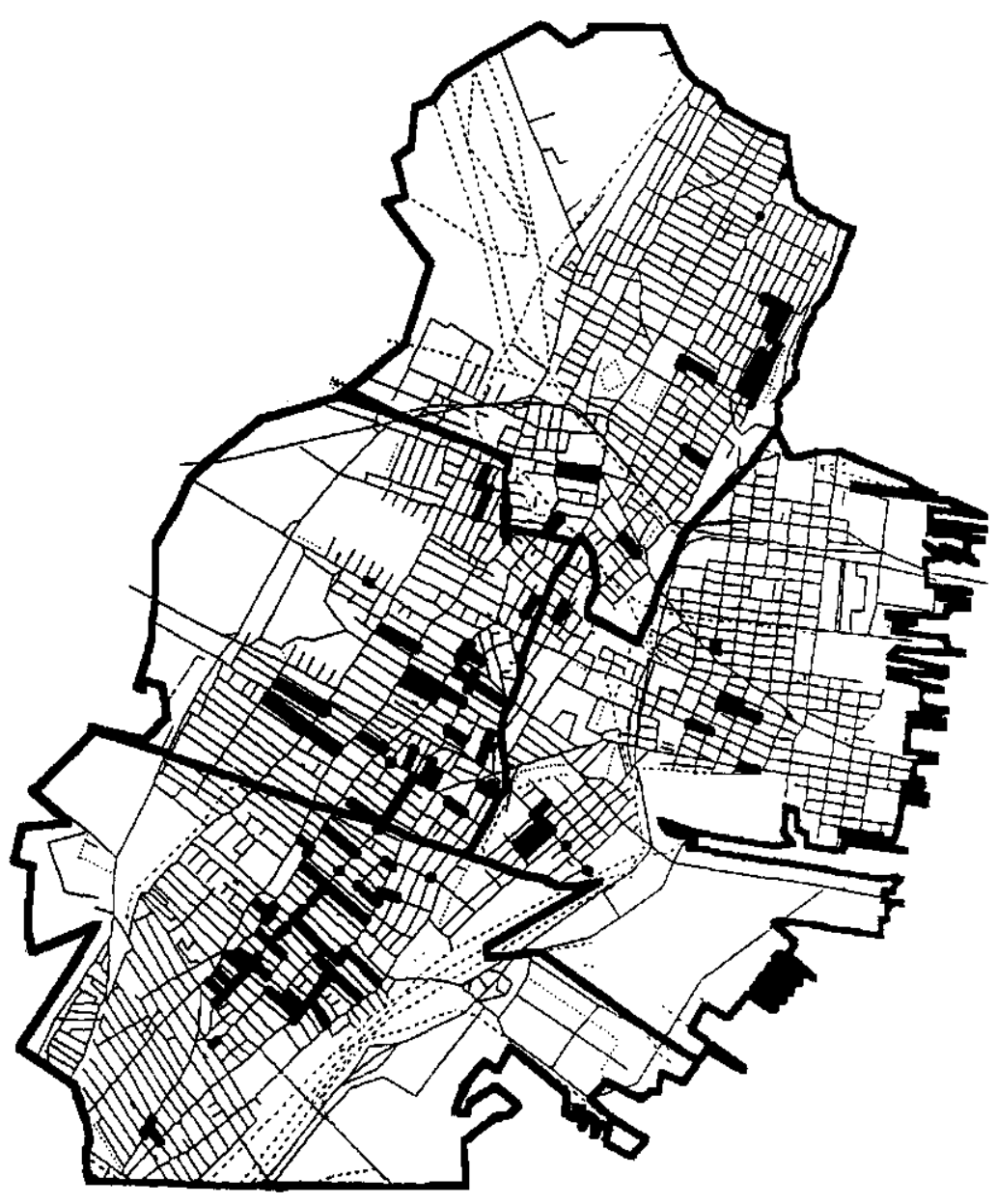




\section{À L'INTÉRIEUR DES MARCHES DE DROGUES}

reconnaissant que les vendeurs traversent rarement des segments de rue et des intersections inactifs afin de participer au commerce des drogues, nous avons relié seulement les segments de rue et les intersections actifs se touchant les uns les autres. En outre, nous avons exclu les zones de marché situées à l'intérieur des limites des projets domiciliaires puisque ces derniers ne sont pas reconnus comme des centres importants de distribution des drogues 9 . Utilisant ces critères, nous avons identifié 56 marchés couvrant 192 carrefours et rues ${ }^{10}$. Le schéma 2 illustre la répartition des marchés.

\section{LES CARACTÉRISTIQUES DES MARCHÉS DE DROGUES DE JERSEY CITY}

Un critère clé utilisé afin d'identifier les marchés de drogues dans la ville de Jersey City fut le type de drogue transigé. Comme l'indique le tableau 1, la majorité des marchés de la ville étaient liés principalement à la vente de cocaïne. Six marchés se spécialisaient essentiellement dans la revente d'héroïne et trois marchés étaient spécialisés dans la marijuana. Dix marchés étaient des centres pour la vente de plus d'une drogue, le plus souvent de la cocaïne et de l'héroïne, ou de la cocaìne et de la marijuana. Les 8 marchés pour lesquels l'information sur le type de drogue transigé n'était pas disponible étaient ceux identifiés par les appels d'urgence et les appels de la ligne info-crime. Presqu'un tiers des marchés identifiés n'englobent qu'une intersection ou un segment de rue. Deux des marchés déployés le long d'une grande série d'îlots d'habitations reliaient plus de dix segments de rue et intersections, formant ainsi les frontières propres à leur marché. Une bonne partie des marchés de drogues identifiés comprend entre deux et quatre intersections ou segments de rue.

9. Les projets domiciliaires furent exclus à la fois parce qu'ils semblaient représenter un type de problème très différent des autres marchés que nous avons identifiés et parce qu'ils reçurent une attention spéciale de la part de la Housing Authority et de sa propre force policière. Huit projets domiciliaires furent exclus de l'étude.

10. Nous avons examiné les commentaires des rapports d'enquête et d'arrestation, et avons appliqué à notre liste un seuil minimum de trois événements par marché. Ces marchés à activité très basse étaient généralement des endroits qui avaient démontré un évênement d'arrestation isolé et qui étaient souvent situé sur la périphérie de marchés plus grands. Nous avons également trouvé que plusieurs "marchés" que nous avions identifiés initialement, étaient en fait des endroits où la police avait établi des achats de stupéfiants et n'étaient donc pas des endroits ayant un problème chronique de drogue. Six marchés comme tels furent exclus de l'étude. 
Tableau 1

Caractéristiques des marchés de drogues

\begin{tabular}{lrr}
\hline Type de drogue transigé & $\mathbf{N}$ & $\%$ \\
\hline Cocaine & 29 & 51,8 \\
Héroine & 6 & 10,7 \\
Marijuana & 3 & 5,3 \\
Deux drogues ou plus & 10 & 17,9 \\
Type de drogue non disponible & 8 & 14,3 \\
Total & $\mathbf{5 6}$ & $\mathbf{1 0 0 , 0}$ \\
\hline Taille des marchés de drogues & $\mathbf{N}$ & $\%$ \\
\hline Un segment de rue ou une intersection & 17 & 30,3 \\
2 - 4 segments de rue et/ou intersections & 23 & 41,1 \\
5 - 9 segments de rue et/ou intersections & 14 & 25,0 \\
10+ segments de rue ou intersections & 2 & 3,6 \\
Total & 56 & $\mathbf{1 0 0 , 0}$ \\
\hline
\end{tabular}

Les données du recensement fournissent un moyen de contraster les marchés de drogues des autres secteurs de la ville: Par exemple, le tableau 2 montre que la valeur médiane moyenne des maisons dans les 56 marchés de drogues était significativement moindre que celle des maisons de Jersey City prise dans son entier. Par contre, un examen plus serré des données montre que le prix des maisons dans les marchés de drogues reflète simplement l'environnement social dans lequel elles se trouvent. Par exemple, le prix des maisons des marchés de drogues des quartiers de la classe moyenne situés au nord et à l'est de la ville illustre une certaine aisance de ces secteurs.

Tableau 2

Caractéristiques des habitations et de la population des aires couvertes par les 56 marchés de drogues par district et pour l'ensemble de la ville

\begin{tabular}{|c|c|c|c|c|c|c|}
\hline & Nord & Sud & Est & Ouest & $\begin{array}{l}\text { L'ensemble } \\
\text { des marchés } \\
\text { de drogues }\end{array}$ & $\begin{array}{l}\text { Ville de } \\
\text { Jersey } \\
\text { City }\end{array}$ \\
\hline \multicolumn{7}{|l|}{ Habitations : } \\
\hline Valeur médiane $(\$)$ & 44254 & 29674 & 63254 & 38312 & 43954 & 62509 \\
\hline \multicolumn{7}{|l|}{$\begin{array}{l}\text { Population : \% de } \\
\text { résidents non-blancs* }\end{array}$} \\
\hline Résidents (district) & 23,0 & 62,5 & 56,5 & 52,0 & ND & 48,5 \\
\hline Résidents (marchés) & 37,6 & 88,4 & 65,5 & 59.8 & 62,8 & ND \\
\hline
\end{tabular}

* Inclut les noirs, les hispaniques, les asiatiques et autres catégories.

Les données du recensement informent aussi sur la composition ethnique des différents quartiers de la ville de Jersey City. Globalement, environ la moitié des résidents de la ville sont non-blancs (ce qui inclut les noirs, 
hispaniques, asiatiques et les autres catégories). Tandis que la proportion de résidents non-blancs vivant au sein des frontières des marchés de drogues est considérablement plus grande que celle observée pour la ville dans son ensemble, les marchés des stupéfiants reflètent à nouveau, jusqu'à une certaine mesure, la composition raciale des communautés dans lesquelles ils se localisent (voir tableau 2). Dans le district nord, principalement blanc, seulement $38 \%$ des résidents vivant à l'intérieur des marchés de drogues ne sont pas de race blanche. Dans le district sud, où plus de $60 \%$ des résidents ne sont pas blancs, presque $90 \%$ de ceux vivant dans les marchés de drogues ne sont pas blancs.

Le nombre d'arrestations pour affaires de stupéfiants et d'appels reçus dans chacun des marchés reflète à la fois le niveau de l'activité des marchés, le niveau des efforts marginaux de la police, et le niveau de délation des citoyens. Parmi ces marchés démontrant une certaine activité d'arrestation pour affaires de stupéfiants, trois d'entre eux ont donné lieu à seulement une ou deux arrestations reliées à la loi sur les stupéfiants. Cinq marchés avaient plus de $\mathbf{S 0}$ arrestations pour cause de narcotiques durant la période examinée de six mois, et trois d'entre eux avaient plus de cent arrestations. Le nombre moyen d'arrestations liées aux stupéfiants par marché (sans antécédent d'arrestation) était de 20. De même, en moyenne, il y avait 19 appels d'urgence reliés aux narcotiques dans chacun des marchés que nous avons étudiés. Deux marchés ont reçu plus de 100 appels dans les six mois précédant le début de l'expérience.

\section{LA CRIMINALITÉ AU SEIN DES MARCHÉS DE STUPÉFIANTS}

Une façon de conceptualiser la concentration spatiale du crime consiste simplement à regarder la proportion de rapports d'infractions spécifiques commises à l'intérieur des surfaces couvertes par le marché de drogues. Nous avons relié les adresses de résidences déclarées se trouvant à l'intérieur des 56 frontières des marchés, avec toutes les arrestations effectuées par le Département de Police de Jersey City entre le $1^{\mathrm{er}}$ janvier 1991 et le 31 décembre 1991, ainsi que tous les appels d'urgence reçus entre le

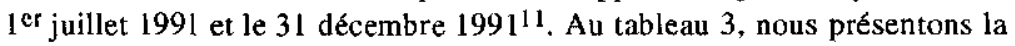
proportion d'arrestations et d'appels répertoriés pour les infractions sélectionnées au sein des marchés de drogues.

11. Nous avons inclu l'information sur les arrestations pour l'année entière afin de fournir une description des arrestations dans la ville de Jersey City aussi détaillée que possible $(\mathrm{N}=15381)$. Nous avons seulement inclu six mois de données sur les appels $(\mathrm{N}=173$ 489) parce que le système de Répartition Assistée par Ordinateur (RAO) n'était pas opérationnel avant le $1^{\text {er }}$ juin 1991 . À cause des biais pouvant possiblement influencer le comportement des policiers lors d'arrestations et d'appels d'urgence, nous utilisons seulement l'information compilée pour la période antérieure au début de l'étape expérimentale du projet. 
Tableau 3

La proportion d'arrestations et d'appels d'urgence pour un certain nombre d'infractions au sein des frontières des marchés de drogues sur l'ensemble de la ville

\begin{tabular}{|c|c|c|c|}
\hline \multicolumn{4}{|l|}{ Arrestations : } \\
\hline Type d'infraction & $\begin{array}{l}\text { Nombre } \\
\text { pour la } \\
\text { ville }\end{array}$ & $\begin{array}{l}\text { Nombre } \\
\text { pour les } \\
\text { marchés }\end{array}$ & $\begin{array}{c}\text { Pourcentage } \\
\text { attribuables } \\
\text { aux marchés }\end{array}$ \\
\hline Stupefiants & 3252 & 1555 & 47,8 \\
\hline Vol qualifié & 521 & 105 & 20,2 \\
\hline Voies de fait & 1821 & 344 & 18,9 \\
\hline Vol-cambriolage & 2735 & 216 & 7,9 \\
\hline Vandalisme & 300 & 30 & 10,0 \\
\hline Possession d'armes prohibees & 410 & 82 & 20,0 \\
\hline Désordre sur la voie publique & 772 & 321 & 41,6 \\
\hline Conduite en état d'ivresse & 273 & 24 & 8,8 \\
\hline Contrefaçon-fraude & 174 & 18 & 10,3 \\
\hline Nuisance publique & 3603 & 143 & 4,0 \\
\hline \multicolumn{4}{|l|}{ Appels d'urgence : } \\
\hline Type d'infraction & $\begin{array}{c}\text { Nombre } \\
\text { pour la } \\
\text { ville }\end{array}$ & $\begin{array}{l}\text { Nombre } \\
\text { pour les } \\
\text { marchés }\end{array}$ & $\begin{array}{l}\text { Pourcentage } \\
\text { attribuables } \\
\text { aux marchés }\end{array}$ \\
\hline Stupéfiants & 3019 & 1420 & 47,0 \\
\hline Contre la personne & 16621 & 2364 & 14,2 \\
\hline Interpersonnel & 14156 & 1696 & 12,0 \\
\hline Propriété & 31015 & 2024 & 6,5 \\
\hline Véhicule-circulation & 26175 & 1729 & 6,6 \\
\hline Nuisance publique & 22050 & 3552 & 16,1 \\
\hline Accidents (feu, explosion...) & 8201 & 940 & 11,5 \\
\hline Moralité publique & 2477 & 492 & 19,9 \\
\hline
\end{tabular}

La plupart des arrestations effectuées à l'intérieur des marchés de drogues l'ont été pour une infraction reliée au trafic ou à la possession de narcotiques, ce qui reflète le processus que nous avons utilisé afin d'identifier les marchés de drogues. Presque la moitié de toutes les arrestations menées dans la ville pour cause de stupéfiants se retrouve au sein des surfaces couvertes par les marchés, lesquels ne représentent pourtant seulement que $4,4 \%$ du nombre total des intersections et segments de rue de la ville $^{12}$. Seule l'infraction de désordre sur la voie publique, une accusation souvent portée et utilisée contre des personnes suspectées de trafic de

12. Il est utile de noter que nous n'avons pas trouvê de différence majeure dans la densité des surfaces entre celles couvertes ou non couvertes par les marchés de drogues. 
drogue lorsqu'une arrestation n'est pas légalement légitime, montre un niveau similaire de concentration.

Les crimes de violence tels que le vol qualifié et les voies de fait, aussi bien que la possession d'armes prohibées, sont aussi des infractions particulièrement fréquentes dans les marchés de drogues. Ici, les marchés de drogues comptent pour un cinquième du total des arrestations pour chacun des crimes, ce qui est plus que quatre fois ce à quoi nous pourrions nous attendre si les arrestations étaient uniformément distribuées à travers toutes les rues et intersections de la ville. Les autres infractions, telles que le cambriolage. le vandalisme et la contrefaçon, arrivent également relativement plus souvent au sein des surfaces couvertes par le marché que dans les autres endroits de la ville. Bien qu'ici les différences ne soient pas aussi importantes en termes absolus, il y a presque deux fois plus d'arrestations rapportées que prévu dans la partie de la ville couverte par les marchés des stupéfiants.

Pour interpréter les données de cette étude, une difficulté majeure surgit du fait que les arrestations pour des crimes autres que ceux reliés aux stupéfiants aient été sur-évaluées à l'intérieur des marchés de drogues simplement parce que ces quartiers recevaient une attention policière plus considérable que les autres. Par exemple, le taux élevé d'accusations pour possession d'armes prohibées enregistré à l'intérieur des frontières des marchés de drogues pourrait être simplement expliqué par une présence policière accrue dans ces secteurs, et il est vraisemblable que la police puisse arrêter une personne pour une infraction liée aux stupéfiants et ensuite, en cours d'arrestation, trouver une arme sur le suspect. En effet. lorsque nous examinons les données des appels d'urgence, lesquelles ne sont pas tellement influencées par les aléas d'une opération policière, nous trouvons une concentration moins grande de crimes au sein des marchés de drogues $^{13}$. Toutefois, il subsiste toujours une sur-représentativité pour les catégories de crime examinées dans ces quartiers. Confirmant à nouveau notre approche initiale, le tableau 3 montre que près de la moitié de tous les appels liés aux stupéfiants furent localisés au sein des marchés de drogues. Dans les cas de vols qualifiés et de voies de fait (définis ici comme des crimes contre la personne), plus de $14 \%$ de l'ensemble des appels furent localisés au sein des marchés de drogues. Cela est plus que trois fois le nombre attendu d'appels compte tenu de la superficie de la ville couverte

13. Les données d'appels d'urgence sont également sujettes à des préjugés, quoique d'un type différent (pour une revue de littérature de ce problème, voir Sherman, Gartin et Buerger, 1989). Les crimes identifiés par un système de répartition téléphonique pourraient représenter des mensonges intentionnels ou une interprétation erronée des événements de la part des victimes, des témoins ou des téléphonistes qui reçoivent l'appel. 
par ces marchés. La concentration d'appels liés aux désordres sur la voie publique et aux atteintes aux bonnes mours est également plus élevée dans les marchés de drogues. Un appel sur cinq concernant les mours, et près d'un appel sur six concernant le désordre sur la voie publique, est relié aux segments de rue ou intersections situés au sein des marchés de drogues ${ }^{14}$.

Une deuxième façon d'examiner la distribution spatiale de la criminalité consiste à identifier la proportion d'un territoire qui a été couvert par la perpétration d'un crime particulier. Dans ce cas, nous ne cherchons pas à établir la proportion globale de criminalité située dans les marchés de drogues, mais plutôt à identifier le nombre d'endroits à l'intérieur d'un marché de drogues où des crimes sont fréquemment commis. Suivant cette méthode, les données du tableau 4 montrent que les marchés de drogues sont des lieux particulièrement dangereux.

Tableau 4

Pourcentage de segments de rue et d'intersections associés à une arrestation ou dे un appel d'urgence pour un cerfain nombre d'infractions

\begin{tabular}{lcc}
\hline $\begin{array}{l}\text { Arrestations : } \\
\text { Type d'infraction }\end{array}$ & $\begin{array}{c}\text { À l'intérieur des } \\
\text { marchés de drogues }\end{array}$ & $\begin{array}{c}\text { À l'extérieur des } \\
\text { marchés de drogues }\end{array}$ \\
Stupéfiants & 80,7 & 9,5 \\
Vol qualifié & 22,3 & 4,7 \\
Voies de fait & 57,8 & 14,7 \\
Vol-cambriolage & 41,4 & 15,7 \\
Vandalisme & 9,9 & 3,7 \\
Possession d'armes prohibées & 23,4 & 4,1 \\
Désordre sur la voie publique & 14,6 & 3,8 \\
Contrefaçon-fraude & 2,1 & 1,1 \\
Nuisance publique & 33,3 & 9,0
\end{tabular}

14. La part disproportionnée d'arrestations et d'appels au sein des marchés de drogues tend à indiquer que les marchés de drogues attirent d'autres activités criminelles. Toutefois, la distribution inégale des marchés de drogues parmi les différents quartjers de Jersey City (voir figure 2) relance la question suivante : le taux global de crimes rapportés au sein des marchés de drogues est-il purement et simplement un reflet d'une distribution de crimes rapportés au travers des différents quartiers de la ville? Si cela s'avère être le cas, nous pourrions espérer que les marchés du district sud, par exemple, puissent montrer un taux plus élevé d'arrestations ou d'appels par segment de rue que ceux du district nord à criminalité plus basse. $L$ 'examen de nos données n'étaye pas cette position. Tandis que les marchés du district nord forment seulement douze pour cent du total des cartrefours et des segments de rue des marchés des stupéfiants, ils comptent pour $23 \%$ des arrestations et pour $17 \%$ des appels. Le district sud, lequel comprend $41 \%$ des intersections et segments de rue, comprend seulement $37 \%$ des arrestations et $35 \%$ des appels générés au sein de l'ensemble des marchés de drogues. 
Tableau 4 (suite)

\begin{tabular}{lcc}
\hline Type d'infraction & $\begin{array}{c}\text { À l'intérieur des } \\
\text { marchés de drogues }\end{array}$ & $\begin{array}{c}\text { À l'extérieur des } \\
\text { marchés de drogues }\end{array}$ \\
Stupéfiants & 85,9 & 14,1 \\
Contre la personne & 95,8 & 55,0 \\
Interpersonnel & 86,9 & 46,7 \\
Propriété & 95,3 & 72,5 \\
Véhicule-circulation & 91,6 & 68,8 \\
Nuisance publique & 96,8 & 59,9 \\
Accidents (feu, explosion...) & 85,9 & 49,8 \\
Moralité publique & 61,9 & 17,1 \\
\hline
\end{tabular}

Tandis que seulement $15 \%$ des rues et des intersections ne faisant pas partie des marchés de drogues ont été le lieu d'une arrestation pour voies de fait ou pour vol durant la période examinée, $60 \%$ des segments de rue et intersections des marchés de drogues ont été le lieu d'une arrestation pour voies de fait, et $40 \%$ le furent pour vol qualifié. Seulement une intersection ou un segment de rue sur vingt ne faisant pas partie du marché est le lieu d'un procès-verbal pour une infraction liée à la possession d'armes prohibées, et seulement un sur vingt-cinq est le lieu d'une arrestation pour vol qualifié. Par contre, 23\% des segments de rue et intersections des marchés de drogues ont été le lieu d'une arrestation pour possession d'armes prohibées et $22 \%$ le furent pour vol qualifié.

Les données provenant des appels d'urgence confirment ces constatations initiales. Par exemple. tandis que seulement un peu plus de la moitié des segments de rue et intersections en debors des marchés de drogues étaient associés à un appel concernant un crime contre la personne durant les six mois examinés, à peu près chaque segment de rue et intersection situé au sein des marchés de drogues était associé à ce type d'appel. Comme prévu, plus de $85 \%$ des endroits situés à l'intérieur des marchés de drogues ont été associés à un appel concernant les narcotiques. Ceci contraste avec le fait que seulement $14 \%$ des segments de rue et intersections situés à l'extérieur des marchés de drogues ont été associés avec un appel d'urgence concernant les narcotiques.

\section{CONCLUSION}

Les zones de la ville de Jersey City couvertes par un marché de drogues comptent pour une part disproportionnée de la criminalité connue. Les intersections et segments de rue situés au sein des marchés de drogues sont également beaucoup plus susceptibles d'être affectés par la criminalité que ces mêmes types d'endroits situés hors des marchés de drogues. De telles constatations tendent à montrer qu'il y a bel et bien un lien drogue- 
crime au niveau des places et des rues. Ainsi, l'idée suivante défendue par Inciardi $(1980: 191)$ au plan individuel : que : « high volume drug use and widespread criminality go hand-in-hand», serait tout aussi valable pour les places et rues.

Par contre, le seul fait que les crimes soient plus fréquents au sein des marchés de drogues ne veut pas automatiquement dire que c'est la drogue elle-même qui produit de tels résultats. En effet, au niveau individuel, ce n'est pas parce qu'un délinquant est aussi un toxicomane qu'il y a nécessairement un lien causal entre les phénomènes; ces deux types de déviance peuvent se développer à partir d'une série de causes communes (voir Altschuler et Brounstein, 1991; Brook et Cohen, 1989) 15 .

Toutefois, l'influence potentielle de la consommation de drogues sur le comportement humain, aussi bien que le contexte social et économique particulier du marché de la drogue, produit une sur-représentation de la criminalité à l'intérieur des marchés de drogues qui n'est pas surprenante. Tandis que les contrevenants refusent souvent d'affirmer que leur consommation de drogues ait entraîné directement leurs actions malheureuses (voir Johnson et al., 1986; Carpenter et al., 1988), certaines drogues auraient un rôle pharmacologique dans la genèse du comportement violent (Goldstein, 1985 ; McBride. 1981 ; Goodman et al., 1986; Felson et Steadman, 1983). En outre, la sur-représentation de la criminalité aux endroits où un grand nombre de vendeurs de drogues sont identifiés ${ }^{16}$ concorde aisément avec l'éclairage que nous apporte la théorie des activités quotidiennes (routine activity approach) sur les comportements criminels (voir Cohen et Felson, 1979). Ainsi, il n'est pas surprenant de constater que les endroits où se concentrent un grand nombre de délinquants et où les drogues sont facilement accessibles soient également des lieux où les crimes violents sont commis.

De plus. il y a de plus en plus d'indices qui nous permettent de croire que le crime fournit un moyen d'assurer le bon fonctionnement du marché de la drogue. Par exemple, Fagan et Chin (1990) ont montré que la plupart des comportements criminels violents commis par les consommateurs de drogue sont reliés au commerce et à la distribution des stupéfiants, plutôt qu'à la seule consommation de ceux-ci. Le crime, ici comme dans d'autres contextes, peut être utilisé comme une stratégie de contrôle social (voir

15. Weisburd et al. (1991) ont montré qu'il n'existe pas de combinaison spécifique de causes expliquant la criminalité des places et endroits. Cependant, ils ont montré que certains types de crimes, comme les vols qualifiés et les voies de fait, sont souvent commis dans des points chauds ( «hors spots») de la ville.

16. Ceci présume, naturellement, que les vendeurs de drogues et leurs clients sont plus susceptibles de participer à des activités criminelles que les non-consommateurs de drogues (voir Altschuler et Brounstein, 1991 ; Collins, 1990). 
Black, 1983: Weisburd, 1984). Le recours au crime peut s'averer être une composante normale et routinière de l'organisation sociale du marché de la drogue. soit dans le but de réduire ou déloger la concurrence, soit comme moyen de résoudre des conflits à propos du prix ou de la qualité des produits.

La discussion évoquée ici est encore quelque peu spéculative. Afin de mieux comprendre pourquoi drogue et crime sont intimement liés dans l'espace, les criminologues auront à reconstituer la scène du marché de la drogue. Les études futures auront à expliquer pourquoi certains endroits deviennent des marchés de drogues et pourquoi certains types de crimes sont plus susceptibles de se produire là plutôt qu'ailleurs. Ici, comme dans d'autres domaines de l'analyse spatiale du crime, les chercheurs devront passer de l'étude des individus déviants à l'étude des places, lieux et endroits déviants. Bien qu'un tel changement nécessite que l'on revise radicalement notre approche du problème criminel, il offre une chance unique de mieux comprendre des aspects importants du problème criminel qui n'ont été qu' effleurés par les études traditionnelles.

\section{BIBLIOGRAPHIE}

ALTSCHULER, D.M. et P.J. BROUNSTEIN (1991), «Patterns of Drug Use, Drug Trafficking, and Other Delinquency Among Inner-City Adolescent Males in Washington D.C. », Criminology, 29 (4), 589-621.

ANNAN, S. (1990), Drug Enforcement in Public Housing, Paper presented at the 1990 annual meeting of the American Society of Criminology, Baltimore, MD.

BLACK, D. (1983), «Crime as Social Control», American Sociological Review, 43. 34-45.

BROOK, J. et P. COHEN (1989), «A Developmental Perspective on Drug Use and Delinquency $m$. In J. McCord (éd.), Facts, Frameworks, Forecasts : Advances in Criminological Theory, vol. 3, 231-251. New Brunswick, N.J., Transaction Publishers.

CARPENTER, C., B. GLASSNER, B. JOHNSON et J. LOUGHLIN (1988), Kids, Drugs, Alcohol and Crime, Lexington, MA, Lexington Books.

CARROLL, P.J. (1989), «Operation Pressure Point: An Urban Drug Enforcement Strategy », FBI Law Enforcement Bulletin, 54 (4), 1-7.

CHAIKEN, J. et M. CHAIKEN (1990), «Drugs and Predatory Crime». In Michael Tonry et James Q. Wilson (éds.), Drugs and Crime, vol. 13, Chicago, University of Chicago Press.

COHEN, L.E. et M. FELSON (1979), «Social Change and Crime Rate Trends : A Routine Activity Approach ", American Sociological Review, 44, 588-608. 
COLLINS, J. (1990), «Summary Thoughts about Drugs and Violence». In M. De La Rosa, E. Lambert et B. Gropper (éds.), Drugs and Violence : Causes, Correlates and Consequences, Rockville, MD, National Institute on Drug Abuse.

ECK, J.E. and W. SPELMAN (1987), "Problem Solving: Problem-Oriented Policing in Newport News", Police Executive Research Forum, Washington, D.C., National Institute of Justice.

FAGAN, J. et K. CHIN (1990), «Violence as Regulation and Social Control in the Distribution of Crack». In Drugs and Violence : Causes, Correlates, and Consequences, édité par M. De La Rosa, E. Lambert et B. Gropper, Rockville, MD, National Institute on Drug Abuse.

FELSON, R.B. et J.J. STEADMAN (1983), «Situational Factors in Disputes Leading to Criminal Violence », Criminology, 21, 59-74.

FERGUSON, H. et P.S. FITZSIMONS (1990), « Drug Abatements : An Effective Tool in the War on Narcotics $»$. The Police Chief, 57, 46-49.

GOLdSTEIN, P. (1985), «The Drugs-Crime Nexus : A Tripartite Conceptual Framework », Journal of Drug Issues, 15, 493-506.

GOODMAN, R.A., J.A. MERCY, F.F. LOYA, M.L. ROSENBERG, J.C. SMITH, N.H. ALLEN, L. VARGAS et R. KOLTS (1986), «Alcohol Use and Interpersonal Violence : Alcohol Detected Homicide Victims", American Journal of Public Health, 76, 144-149.

GREEN, L. (1993), Treating Deviant Places : A Case Study Examination of the Beat Health Program in Oakland, California, Unpublished Doctoral Dissertation, Rutgers University, Newark, N.J.

HAYESLIP, D.W., Jr (1989), Local-Level Drug Enforcement : New Strategies (Report $n^{\circ} 213$ ), Washington, D.C., National Institute of Justice.

HILLSMAN, S. (1992), The Community Effects of Street Level Narcotics Enforcement : A Study of the New York City Police Department's Tactical Narcotics Teams, An interim report, Vera Institute.

INCIARDI, J.A. (1980), «Youth, Drugs, and Street Crime». In F. Scarpitti et S.K. Datesman (éds.), Drugs and Youth Culture, Beverly Hills, Sage Publications.

INNES, C. (1988), Drug Use and Crime, Washington, D.C., U.S. Department of Justice.

JOHNSON, B.D., E. WISH, J. SCHMEIDLER et D.H. HUIZINGA (1986), «The Concentration of Delinquent Offending: Serious Drug Involvement and High Delinquency Rates\%. In B.D. Johnson et E. Wish (éds), Crime Rates Among Abusing Offenders, New York, Interdisciplinary Research Center, Narcotics and Drug Research, Inc.

KENNEDY, D. (1993), Closing the Market: Controlling the Drug Trade in Tampa, Florida, National Institute of Justice - Program Focus, U.S. Department of Justice, Washington, D.C.

KLEIMAN, M.A. et K.D. SMITH (1990), «State and Local Drug Enforcement: In Search of a Strategy». In M. Tonry et J.Q. Wilson (éds.), Drugs and Crime, Chicago, University of Chicago Press.

MCBRIDE, D. (1981), «Drugs and Violence». In J. Inciardi (£d.), The Drugs Crime Connection, Beverly Hills, Sage Publications.

MALTZ, M., A. GORDON et W. FRIEDMAN (1991), Mapping Crime in its Community Setting : Event Geography Analysis, New York, Springer-Verlag. 
SHERMAN, L.,P. GARTIN et M. BUERGER (1989), «Hot Spots of Predatory Crime $\gg$, Criminology, 27 (1), 27-56.

U.S. DEPARTMENT OF JUSTICE (1992), Drugs, Crime, and the Justice System : A National Report from the Bureau of Justice Statistics, Washington, D.C., U.S. Government Printing Office.

WEISBURD, D. (with V. VINITZKI) (1984), "Vigilantism as Rational Social Control : The Case of the Gush Emunim Settlers », Political Anthropology, vol. 4, 69-87.

WEISBURD, D. et L. GREEN (1993), * Defining the Street Level Drug Market ». In D.L. MacKenzie and C. Uchida (éds.), Drugs and the Criminal Justice System: Evaluating Public Policy lnitiatives, Newbury Park, CA, Sage Publications.

WEISBURD, D., L. MAHER et L. SHERMAN (1991), «Contrasting Crime General and Crime Specific Theory: The Case of Hot Spots of Crime», Advances in Criminological Theory, vol. 5. 